\title{
Concurrent salmonellosis and histoplasmosis in AIDS: an unusual co-existence in Britain
}

\author{
S Crowley, R J Coker, S M Murphy
}

\begin{abstract}
A patient is described with systemic salmonellosis which was unresponsive to therapy. Histoplasma capsulatum was isolated after death and we suggest that dysfunction of the reticuloendothelial system by $H$. capsulatum may have altered the prognosis.
\end{abstract}

\section{Introduction}

Both disseminated Histoplasma capsulatum and non-typhi salmonella bacteraemia are manifestations of immunosuppression in patients infected with human immunodeficiency virus (HIV) and are AIDS-defining diagnoses. In the non-immunocompromised salmonella bacteraemia occurs in $5 \%$ of patients with infection, but rates of $40 \%$ to $80 \%$ are reported in the immunocompromised ${ }^{12}$ and invasive infection requires prompt antibiotic therapy. Post-treatment relapse in the immunocompromised remains a problem and long-term suppressive therapy is indicated. ${ }^{34}$

Histoplasmosis was initially reported in patients with AIDS in 1982 though it had previously been recognised as an opportunistic pathogen in those with defective cellular immunity. Nearly all cases associated with AIDS are disseminated, and it is often the first opportunist infection in endemic areas. In one report from Houston histoplasmosis was the first opportunist infection in $75 \%$ of cases of AIDS. ${ }^{5}$ In the UK, however, histoplasmosis in AIDS is attributed to reactivation of disease contracted in endemic areas and is therefore infrequent.

Involvement of the reticuloendothelial system with macrophages full of yeast cells is a pathological hallmark in histoplasmosis. It has been suggested that, as a result of macrophage dysfunction, an impaired ability to kill intracellular pathogens such as salmonella may occur. ${ }^{6}$ Dual infection with Histoplasma and salmonella has been reported in only two patients with AIDS previously, both of whom were from the U.S. ${ }^{6}$

Department of
Genitourinary
Medicine, Patrick
Clements Clinic,
Central Middlesex
Hospital, Acton Lane,
London NW10 7NS,
UK
S Crowley
R J Coker
S M Murphy
Address for correspondence:
Dr Coker.
Accepted for publication
11 March 1992

\section{Case report}

A 26 year old HIV-antibody positive homosexual Brazilian man presented with a 3 month history of diarrhoea, intermittent fever, and weight loss. Two weeks prior to admission he noted a facial skin rash, dry cough and dyspnoea. He had been an infrequent attender to outpatients and had declined zidovudine therapy. His $\mathrm{T}$ cell subset count 8 months prior to admission was $194 / \mathrm{mm}^{3}$. He had not recently travelled to South America.
On examination he was pyrexial $\left(38.6^{\circ} \mathrm{C}\right)$, cachexic, and anaemic. He had generalised lymphadenopathy, pustular facial rash, and oral candidosis.

Initial investigations revealed: $\mathrm{Hb} 8.8 \mathrm{~g} / \mathrm{dl}$, WBC $2.0 \times 10^{9} /$, total lymphocyte count 0.2 $\times 10^{9} /$, platelet $130 \times 10^{9}$; Chest radiograph was normal; liver function tests were normal, except albumin $15 \mathrm{~g} / \mathrm{l}$; mid-stream urine culture sterile; stool cultures negative; blood cultures grew $S$. typhimurium after 36 hours.

Treatment was commenced with ciprofloxacin $(200 \mathrm{mg}$ b.d. intravenously for 24 hours and subsequently $750 \mathrm{mg}$ b.d. orally). He remained unwell and pyrexial, and continued to deteriorate. Blood cultures were sterile after 4 days and remained so until death.

Histoplasma antigen complement fixation test and immunodiffusion tests were negative. A skin biopsy performed shortly before death grew $H$. capsulatum on culture, the result not being known until after death; histological examination was unhelpful.

He died 18 days after admission. Post mortem examination confirmed the presence of yeast-like organisms consistent with $H$. capsulatum in macrophages in the tongue, testes, skin, spleen, lymphnodes and the reticuloendothelial cells of the liver.

\section{Discussion}

The clinical features of disseminated histoplasmosis are nonspecific, most patients presenting with symptoms of fever and weight loss. The infection usually begins in the lungs, probably as a result of reactivation, though pulmonary features may not be present, and the chest radiograph may be normal as in this case. When untreated severe clinical manifestations resembling septicaemia may ensue, and as patients are often concurrently infected with other opportunist pathogens a careful diagnostic search is required. Cutaneous manifestations, including pustular, follicular, maculopapular, and erythematous lesions, occur in up to $17 \%$ of cases.

Since the prognosis is better if treatment is begun early it is important to establish the diagnosis rapidly. Isolation of $H$. capsulatum in cultures from a variety of body tissues proves the diagnosis in most cases, and histopathologic staining of tissue sections or peripheral blood smears with Gomori methenamine silver provides a means for rapid diagnosis. Anti- $H$. capsulatum antibody detection by immunodiffusion or complement fixation also has a role with sensitivity approaching $7 \%{ }^{7}$ 
The coexistence of disseminated histoplasmosis in association with salmonellosis in this case, may, as a result of impaired macrophage function, have prevented antibacterial therapy from being effective. Wheat et $a l^{6}$ suggested that reticuloendothelial "blockade" may result from this intracellular yeast. "Blockade" of the reticuloendothelial system is characteristic of sickle cell anaemia, bartonellosis, and louseborne relapsing fever and serious Salmonella infection occurs in these diseases.

Ampicillin, chloramphenicol and co-trimoxazole are effective in invasive non-typhi salmonellosis, but the lack of bactericidal activity against Salmonella spp at clinically achievable levels, and the toxicity of chloramphenicol and co-trimazole, particularly in those infected with HIV, constrain their use. ${ }^{8}$ The quinolones are active against salmonella, with excellent intracellular penetration and concentrations within neutrophils and macrophages. ${ }^{9}$ Ciprofloxacin has been used with success in immunocompromised patients ${ }^{10}$ and hence its choice in the case described.

This case highlights the need for clinicians to maintain their awareness of the possible coexistence and interaction of pathogens in AIDS patients. When salmonellosis is diagnosed and there is a poor response to treatment disseminated histoplasmosis should also be con- sidered. Treatment with amphotericin B is highly effective in histoplasmosis and may, in this case, have altered the outcome.

1 Jacobs JL, Gold JWM, Murray HW, Roberts RB, Armstrong D. Salmonella infections in patients with the acquired immunodeficiency syndrome. Ann Intern Med 1985;102:186-8.

2 Chaisson RE. Infections due to encapsulated bacteria, salmonella, shigella, and campylobacter. In: Sande MA, Volberding PA, eds. The Medical Management of AIDS. Philadelphia, PA, WB Saunders 1990:247-58.

3 Jacobson MA, Hahn SM, Gerberding JL, Lee B, Sande MA. Ciprofloxacin for salmonella bacteremia in the acquired immunodeficiency syndrome (AIDS). Ann Intern Med 1989;110:1027-9.

4 Sperber SJ, Schleupner CJ. Salmonellosis during infection with human immunodeficiency virus. Rev Infect Dis 1987;9:925-34.

5 Johnson PC, Khardori N, Najiar AF, Butt F, Mansell PWA, Sarosi GA. Progressive disseminated histoplasmosis in Sarosi GA. Progressive disseminated histoplasmosis in patients with acquired

6 Wheat LJ, Rubin RH, Harris NL, et al. Systemic salmonellosis in patients with disseminated histoplasmosis. Arch Intern Med 1987;147:561-4.

7 Wheat LJ, Connoly-Stringfield PA, Baker RL, et al. Disseminated histoplasmosis in the acquired immune deficiency syndrome: clinical findings, diagnosis and treatment, and review of the literature. Medicine (Baltimore) 1990;69:361-74.

8 Gordin AJ, Simon GL, Wofsy CB, Mills J. Adverse reactions to trimethoprim-sulfamethoxazole in patients with the acquired immunodeficiency syndrome. Ann Intern Med 1984;100:495-9.

9 Easmon CSF, Crane JP, Blowers A. Effect of ciprofloxacin on intracellular organisms: in-vitro and in-vivo studies. $\mathcal{F}$ Antimicrob Chemother 1986;18, Suppl. D:43-8.

10 Zumler A, Lewis D, Brown J. Ciprofloxacin treatment of recurrent Salmonella typhimurium septicaemia in a splenectomized and immunosuppressed patient. $f$ Antimicob Chemother 1988;21:809-10. 